\title{
The Dynamic Analysis of Tension Stringing in Transmission Lines Based on ADAMS
}

\author{
XIAO Qi ${ }^{1, a}$, ZHAO Hailing ${ }^{1, b^{*}}$, LU Shuai ${ }^{2, c}$ \\ ${ }^{1}$ Northeast Dian Li University, China \\ ${ }^{2}$ Shandong Taikai Transformer Ltd., China

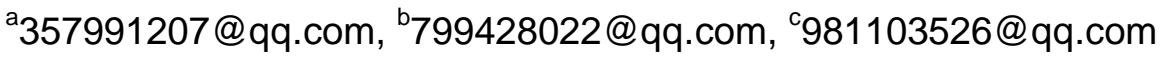

\begin{abstract}
Keywords: Transmission lines, Tension stringing system, ADAMS virtual prototyping, Overhead conductor, Traction rope, Dynamic tension.
\end{abstract}

\begin{abstract}
In recent years, with the power grid and ultra-high voltage power transmission project increased, tension stringing is taking an important part in transmission project. Due to the shortcomings of traditional theoretical formulas, it is not precisely to reflect the dynamic tension of wires. In this paper, the model of the tension stringing construction has been completed. And the time of simulation was reduced by taking the advantage of ADAMS, secondary development function of software and the simplified model. By extracting several sets of axial tension of overhead conductor and traction rope, we can analyze the dynamic characteristics of tension stringing wires. According to the simulation results, we can get the time and the location that wires and traction rope became maximum tension. Finally, the results of hand calculated were compared with the simulation results, which verified the correctness of the model. Based on the above results, analyzed and summarized the dynamic tension stringing of the wires. And the results can give the reasonable advice and practical reference for the projects.
\end{abstract}

\section{Introduction}

In recent years, with the rapid development of Chinese economic construction, the demand for electricity has been increasing, which made the power industry become the pillar of the country's economy [1]. As the construction of overhead transmission lines is getting into a new era, the construction technology wired from human unfolding has been gradually transforming into full mechanization of construction [2]. But it may cause a waste of resources or lack of potentially dangerous judgment during the tension stringing process, if the engineering used the artificial parameters of the partial calculations.

The dynamic tension stringing simulation system was built by using the secondary development function of ADAMS software and a reasonable simplification of the model [3]. And finally the hand calculated results of the overhead conductor tension were compared with the simulation results, which verified the correctness of the model.

By extracting the axial tension of overhead conductor and traction rope, we could analyze the dynamic characteristics of the process of tension stringing wires and traction rope. According to the simulation results, we can get the time and location that wires and traction rope were in maximum tension. Finally, summarized and studied the tension stringing process and gave reasonable advice practical reference for the project.

\section{Tension Stringing System Model}

The Model of Pulley and Traction Broad. Traction equipment provides traction and braking tension for the system of tension stringing. The lines are put on the entire line successively through pulleys on the towers. In the process of unfolding, wires cross through the pulleys for several times and stay a period of time before the next step. Wires move back and forth in tackle under the influence 
of wind. When through the pulleys, wires are bent and stretched, and it produces friction between the interlayer and stocks. Wires and ropes are connected by traction board, which would make wires simultaneously unfolded. ADAMS software provides Pro/E with seamless interface mech/pro. With the powerful modeling and assembly functions of Pro/E [4], three lines tackle geometric model was established, shown in Fig. 1; one pulling two traction board geometry model was shown in Fig. 2.

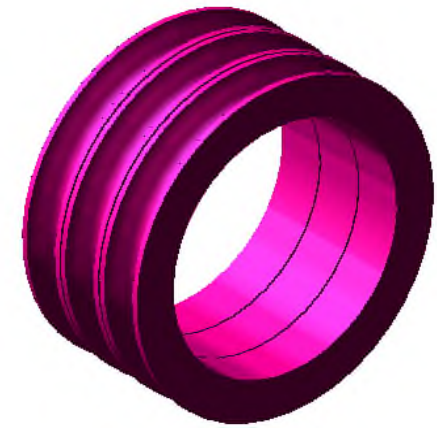

Fig. 1 The geometric model of pulley.

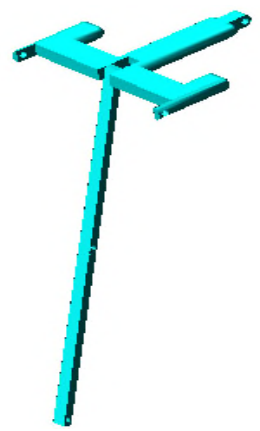

Fig. 2 The geometric model of traction board.

The Model of Wires. The model of wires took a very important part in the entire simulation of the whole modeling, so it was necessary to choose the appropriate modelling way based on the characteristics of the model. According the method of approximately using a rope instead of a rack and pinion described in literature [5], we can get a relatively good simulation speed, but we cannot make the rope wind the requirements. In literature [6], ADAMS/cable model could easily set the model parameters and modeling types, so it is quick and easy to build rope pulley system model. But for the purposes of this model, ADAMS/cable module cannot meet the requests. In contrast, the most suitable modeling method is currently bushing [7-8] connection model, which would lead wires and traction rope to a number of discrete small cylinders, and then matches with the traction board. Finally, contact was added between the wires and pulleys. In the paper bushing considered the character of ropes well, such as stretch, shear, torsion and other characteristics.

There are schematic diagram that added flexible force by defining six parts to two small cylinders, as shown in Fig. 3.

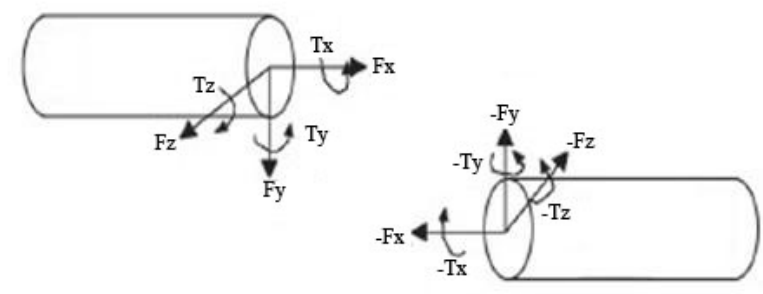

Fig. 3 The force between two cylindrical micro-segments.

The bushing equation is as follows:

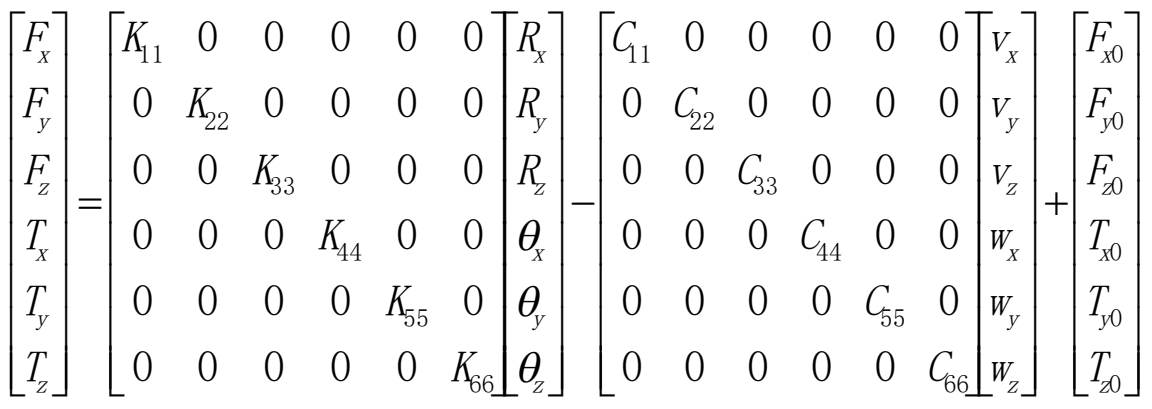


where, $R_{i}$-relative displacement between I-Marker and J-Marker coordinate system; $\theta_{i \text { - relative }}$ angular displacement between I-Marker and J-Marker coordinate system; $V_{i}$-relative velocity between I-Marker and J-Marker coordinate system; $W_{i}$-relative angular velocity between I-Marker and J-Marker coordinate system; $F_{i 0}$-the initial force; $\mathrm{T}_{\mathrm{i} 0}$-the initial torque; $K$-stiffness coefficient; $C$-damping coefficient $[8,9]$.

The counterforce of bushing is as follows:

$$
F_{i}=-F_{j}
$$

$$
\mathrm{Ti}=-\mathrm{Tj}-\delta \mathrm{Fj}
$$

What can be seen from the above equations was that the flexible force acting on the two small micro-cylindrical segments was related to the relative displacement, the relative angular displacement, relative velocity and relative angular velocity. In order to make the deformation, vibration characteristics and the dynamics of wires and ropes match the realistic projects; we should determine the relevant parameters.

Tensile, shear, torsional and bending stiffness coefficient are calculated as follows:

$$
\left\{\begin{array}{l}
K_{11}=\frac{E A}{L} \\
K_{22}=K_{33}=\frac{G A}{L} \\
K_{44}=\frac{G \pi d^{4}}{32 L} \\
K_{55}=K_{66}=\frac{E I}{L}=\frac{E \pi d^{4}}{64 L}
\end{array}\right.
$$

where, $E$-elastic modulus of wires; $G$-Shear modulus of wires; $A$-cross-sectional area of wires; $L$-the length of two small micro-cylindrical segments; $I$-moment of inertia; $D$-diameter.

This model was of tension stringing, wherein a total of two conductors, respectively lengthened out to 320 meters; the pulling rope was 282 meters. Traction rope and wires were made of different diameter cylindrical section, a total of 922 segments, 919 bushings force. Torsion damping was the greater factor, which was selected from between 1-10 according to the actual situation of the project [10]. Because of too many model parts, so the model of wires was completed by ADAMS/View command language. The model of tension stringing system was shown in Fig. 4.

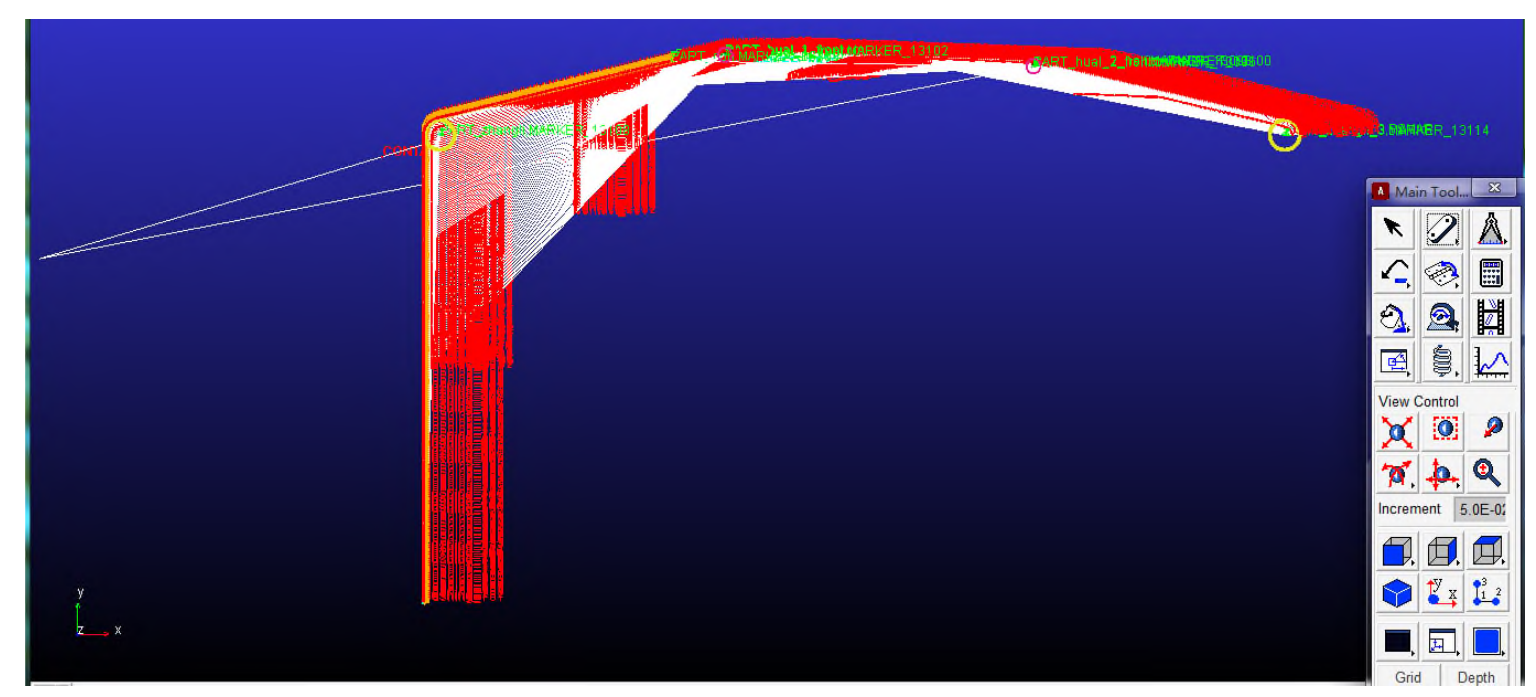

Fig. 4 The model of tension stringing system. 
Contact Applied and Simulation Speed Optimizations. In the actual project, wires and traction broad are in contact with the pulleys. It cannot be ignored that pulleys attrited the wires and the collision between the traction board and the pulleys. However, contact force was the main reason which leads to time-consuming of simulation [11]. Therefore, in order to reduce the consumption of simulation, it was necessary to minimize the amount of contact force, which may increase the possibility of success of the simulation. To improve the simulation speed, there were three methods in this paper, such as simplifying the tension stringing system model, controlling the number of contact force and dynamically activating or failing the contact [12]. In view of the contact force is exerted on the two parts, if separated from each other, it would not produce the contact force. So during the simulation, we can roughly determine the position where the contact force was zero according to calculating. It would greatly improve the speed of simulation by making ADAMS/Solver scripts to dynamically reduce the number of contact force.

\section{Verify the Model of Tension Stringing System}

Theoretical Calculation of Axial Tension. In any tension stringing diagrams, the bellow formulas are used to calculate the axial tension. The formulas related to axial tension, traction and tension are as follows.

The axial tension of the rope at left side of the $\#_{i}$ tower:

$$
\mathrm{T}_{\mathrm{i}}=\mathrm{m \varepsilon} \varepsilon_{0}^{\mathrm{i}-2}\left(\mathrm{~T}_{\mathrm{T}}+\mathrm{wh}_{1} / \cos \varphi_{1}\right)+\mathrm{w}_{0}\left( \pm \varepsilon_{0}^{\mathrm{i}-2} \mathrm{~h}_{2} / \cos \varphi_{2} \pm \cdots \pm \mathrm{h}_{\mathrm{i}} / \cos \varphi_{\mathrm{i}}\right)
$$

The axial tension of the rope at right side of the ${ } i$ tower:

$$
T_{i}^{\prime}=m \varepsilon \varepsilon_{0}^{i-1}\left(T_{T}+w h_{1} / \cos \varphi_{1}\right)+w_{0}\left( \pm \varepsilon_{0}^{i-1} h_{2} / \cos \varphi_{2} \pm \cdots \pm h_{i} \varepsilon_{0} / \cos \varphi_{i}\right)
$$

The axial tension of the wire at left side of the ${ }^{\#} i$ tower:

$$
T_{i i}=m \varepsilon^{i-1} T_{T}+m w\left(\varepsilon^{i-1} h_{1} / \cos \varphi_{1} \pm \varepsilon^{i-2} h_{2} / \cos \varphi_{2} \pm \cdots h_{i} / \cos \varphi_{i}\right)
$$

The axial tension of the wire at right side of the ${ }_{i} i$ tower:

$$
T_{i i}^{\prime}=m \varepsilon^{i} T_{T}+m w\left(\varepsilon^{i} h_{1} / \cos \varphi_{1} \pm \varepsilon^{i-1} h_{2} / \cos \varphi_{2} \pm \cdots \pm \varepsilon h_{i} / \cos \varphi_{i}\right)
$$

where, $T_{T}$-axial tension of each wire; $m$-the number of wires; $w$-weight of one meter wire; $w_{0}$ - weight of one meter rope; $\varepsilon, \varepsilon_{0}$-drag coefficient of wire and rope, $1.015 ; \varphi_{i}$-elevation angle of two suspension points; $h_{i}$-elevation of two suspension points.

The Analysis of Axial Tension in Model. After the simulation, extracted any small micro-cylindrical wires segments to verify whether the axial velocity matched the actual velocity. Axial velocity of traction rope was shown in figure 5. It can be drawn from the following figure, the axial velocity of traction rope met the requirements, and there was an obviously fluctuation at the instant moment of beginning. As time increased, pulling speed became stable at speed $1 \mathrm{~m} / \mathrm{s}$. At the beginning of traction, it was necessery to appropriately reduce the pulling speed when near the towers.

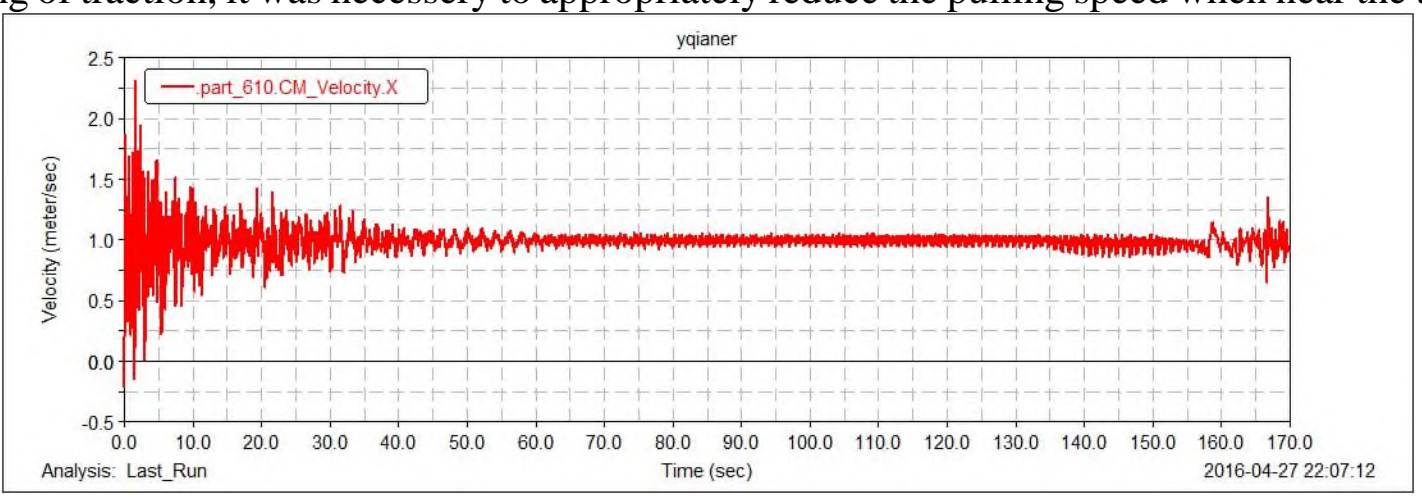

Fig. 5 The axial speed of traction rope. 
Finally, verifted the axial tension of wires whether met engineering requirements. Firstly, according to the axial tension theoretical formulas to calculate the left and right axial tension of wires and traction rope besides ${ }^{\#} 1,{ }_{2} 2$ towers. Next, extracted bushing force of small cylindrical micro-segment in ADAMS/Postprocessor. Then compared the value of the theoretical formula and dynamics simulation results. Extracted bushing499 and 509 of traction rope and bushing149 and 189 of wires. The bushing of traction rope and the bushing of wire were respectively as follows.

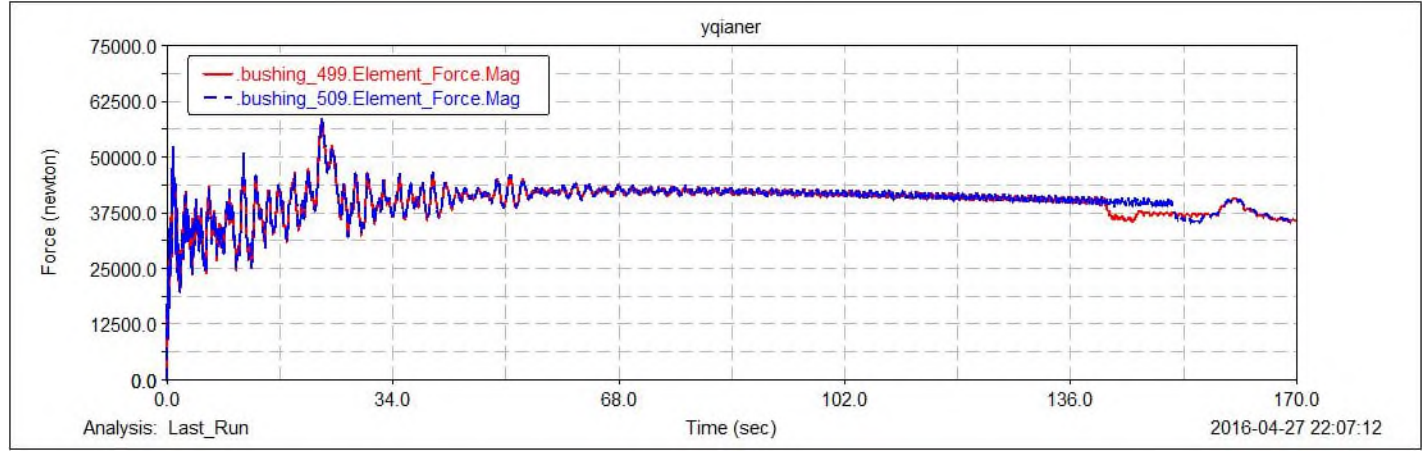

Fig. 6 Bushing 499 and 509 of traction rope.

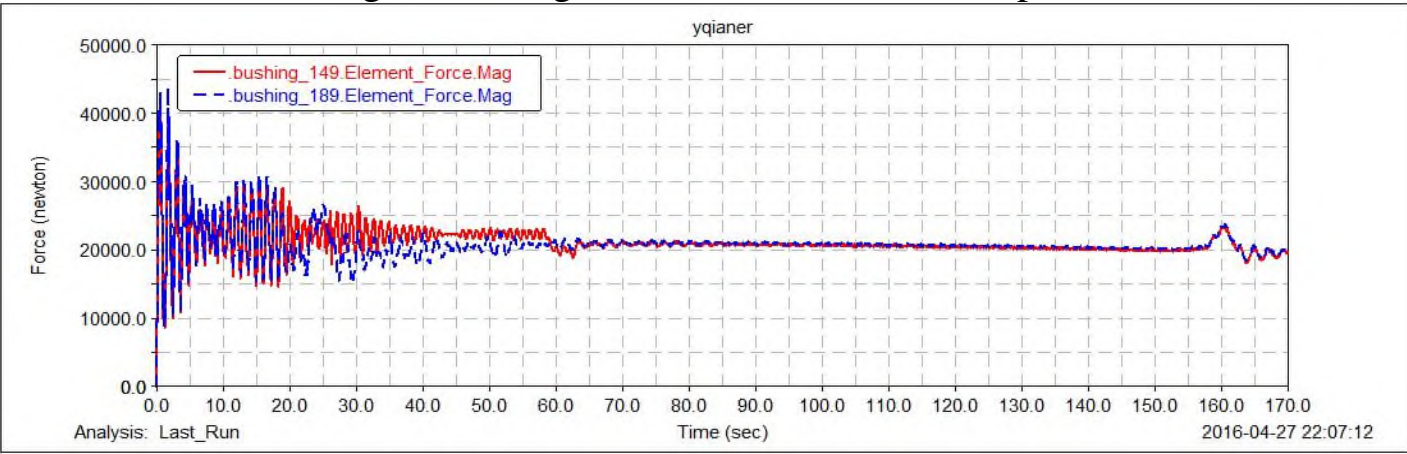

Fig. 7 Bushing 149 and 189 of wire.

From the above figures, we can get dynamic change of tension and the time of the maximum axial force point, such as the bushing 509 reached $43.08 \mathrm{KN}$ at $30.2 \mathrm{~s}$, and we can also find the regular pattern of fluctuations. Through a comprehensive view of the simulation system, it could be found that the bushing force of wires near the pulleys fluctuated obviously. Because of the weight of wires and the long distance, the required traction force and braking tension should correspondingly increase. In the initial stage $0-10$ s of the simulation, dynamic tension fluctuation was highly.

Compared axial force with the theoretical value, the error was in the range of about $5 \%$,which verified the model correctly. Comparison of theoretical values and simulation values was shown in Table 1.

Table 1. Theoretical values and simulation values.

\begin{tabular}{c|c|c|c}
\hline Tension & Theoretical values $/ \mathbf{K N}$ & Simulation values /KN & Error \% \\
\hline$T_{1}$ & 40.35 & $\mathbf{3 8 . 0 6}$ & $\mathbf{5 . 7 0}$ \\
\hline$T_{1}^{\prime}$ & 40.95 & 39.89 & $\mathbf{2 . 6 0}$ \\
\hline$T_{2}$ & 40.23 & 39.55 & $\mathbf{1 . 7 0}$ \\
\hline$T_{2}^{\prime}$ & 40.83 & 43.08 & $\mathbf{5 . 5 0}$ \\
\hline$T_{11}$ & $\mathbf{2 0 . 1 7}$ & $\mathbf{1 8 . 5 6}$ & $\mathbf{7 . 9 8}$ \\
\hline$T_{22}^{\prime}$ & $\mathbf{2 0 . 6 9}$ & $\mathbf{2 1 . 7 5}$ & $\mathbf{5 . 1 2}$ \\
\hline
\end{tabular}

According to the Table 1, it can be obtained that the error between theoretical value and simulation value was controlled at about $5 \%$, which verified the correctness of the model.

So the value of simulation model had a certain reliability, which can provide references guidance for engineering. 


\section{Summary and Conclusions}

This paper was based on the $1000 \mathrm{KV}$ transmission line engineering, selecting the cross section as the research object. In the paper, the whole model of tension stringing was built by the software of ADAMS.

The main conclusions are as follows:

(1) It can be quickly to build the modle by using micro language and the secondary development function of the software. To improve the simulation speed, the tension stringing system model was simplified by controlling the number of contact force and dynamically activating or failling the contact force.

(2) Through the simulation model, we can fully observe the whole process of the tension stringing, which can reflect the dynamic characterastic of wires. We can also obtain the precise size and location of tension during each period of time, which could conveniently better guide engineering practice.

(3) In this paper, the effect of wind speed on the simulation was not considered, which would lead to the diffrence between the simulation and the engineering practice. It is necessery to make a more futher research on the topic.

(4) In the next research, the paper would consider the impact between the traction board and the pullys. It would be a worthy issue to study how to slove the problem.

\section{References}

[1] J. C. Zheng, The Analysis of Power Technology Trends, J. Power Syst. Technol. 21(11) (1997) 4-10.

[2] Geller, Jeffrey, Tornetta, Paul III, Tiburzi, etal, Tension Wire Position for Hybrid External Fixation of the Proximal, Tibia. September/October - 14(7) (2000) 455-52.

[3] S. E. Andrew, Efficient Modeling of Extensible Cables and Pulley Systems in Adams, In Europe Adams Conference, London, 2002, 1-10.

[4] D. Su, H. M. Zhao, The integrated modeling method of driving axle, J. Mech. Des. Manufact. 1 (2010) 235-236.

[5] W. G. Yang, T. D. Guo, The Non-uinform Property of Energy Consumption and its Solution to the Wireless Sensor Network, J. Educ. Technol. Comput. Sci. (ETCS), 2 (2010) 186-192.

[6] C. W. Tian, J. X. Ma, J. Wang, Rope Modeling Method Based on ADAMS, J. China CSEE, 27(29) (2007) 108-112.

[7] D. X. Wang, L. Yin, Y. Li, The model of rope and dynanic simulation, J. Min. Mach. 38(8) (2010) 20-23.

[8] Z. Y. Ge, C. B. Yin, Bridge crane crash safety simulation, J. Ind. Saf. Environ. Protect. 8 (2015) $39-42$.

[9] W. Zhou, J. J. Yi, J. R. Zhen, A modeling method of ADAMS software, J. Mod. Manuf. Eng. 5 (2004) 38-39.

[10]E. M. Abdel- Rahman, A. H. Nayfeh, Cargo- Pendulation Reduction in Boom Cranes via Cable-Length Manipulation Proceeding of the Structures, Structural Dynamics, and Materials Conference and Exhibit, C. 41st AIAA, Atlanta, GA, 2000: 3-61.

[11] A. P. Li, C. P. Jiang, Dynamic tension simulation based on ADAMS, J. Mod. Manuf. Eng.1 (2010) 43-46.

[12] S. R. Yan, B. C. Wen, Computer simulation of lifting rope tension, J. Min. Mach. (3) (1998) 4-6. 